\title{
BMJ Open Identification of anatomic risk factors for acute coronary events by optical coherence tomography in patients with myocardial infarction and residual nonflow limiting lesions: rationale and design of the PECTUS-obs study
}

\author{
Jan-Quinten Mol (1) , ${ }^{1}$ Anouar Belkacemi, ${ }^{2}$ Rick HJA Volleberg, ${ }^{1}$ \\ Martijn Meuwissen, ${ }^{3}$ Alexey V Protopopov, ${ }^{4}$ Peep Laanmets, ${ }^{5}$ Oleg V Krestyaninov, ${ }^{6}$ \\ Robert Dennert, ${ }^{7}$ Rohit M Oemrawsingh, ${ }^{8}$ Jan-Peter van Kuijk, ${ }^{9}$ Karin Arkenbout, ${ }^{10}$ \\ Dirk J van der Heijden, ${ }^{11}$ Saman Rasoul, ${ }^{12,13}$ Erik Lipsic, ${ }^{14}$ Steven Teerenstra, ${ }^{15}$ \\ Cyril Camaro, ${ }^{1}$ Peter Damman, ${ }^{1}$ Maarten $\mathrm{AH}$ van Leeuwen (D) ${ }^{2}$ \\ Robert-Jan van Geuns, ${ }^{1}$ Niels van Royen ${ }^{1}$
}

To cite: Mol J-Q, Belkacemi A, Volleberg RHJA, et al. Identification of anatomic risk factors for acute coronary events by optical coherence tomography in patients with myocardial infarction and residual nonflow limiting lesions: rationale and design of the PECTUS-obs study. BMJ Open 2021;11:e048994. doi:10.1136/ bmjopen-2021-048994

- Prepublication history for this paper is available online. To view these files, please visit the journal online (http://dx.doi. org/10.1136/bmjopen-2021048994).

Received 12 January 2021 Accepted 16 June 2021

Check for updates

(c) Author(s) (or their employer(s)) 2021. Re-use permitted under CC BY-NC. No commercial re-use. See rights and permissions. Published by BMJ.

For numbered affiliations see end of article.

Correspondence to

Professor Niels van Royen; niels.vanroyen@radboudumc.nl

\section{ABSTRACT}

Introduction In patients with myocardial infarction, the decision to treat a nonculprit lesion is generally based on its physiological significance. However, deferral of revascularisation based on nonischaemic fractional flow reserve (FFR) values in these patients results in less favourable outcomes compared with patients with stable coronary artery disease, potentially caused by vulnerable nonculprit lesions. Intravascular optical coherence tomography (OCT) imaging allows for in vivo morphological assessment of plaque 'vulnerability' and might aid in the detection of FFR-negative lesions at high risk for recurrent events.

Methods and analysis The PECTUS-obs study is an international multicentre prospective observational study that aims to relate 0 CT-derived vulnerable plaque characteristics of nonflow limiting, nonculprit lesions to clinical outcome in patients with myocardial infarction. A total of 438 patients presenting with myocardial infarction (ST-elevation myocardial infarction and non-ST-elevation myocardial infarction) will undergo OCT-imaging of any FFR-negative nonculprit lesion for detection of plaque vulnerability. The primary study endpoint is a composite of major adverse cardiovascular events (all-cause mortality, nonfatal myocardial infarction or unplanned revascularisation) at 2-year follow-up. Secondary endpoints will be the same composite at 1-year and 5-year follow-up, target vessel failure, target vessel revascularisation, target lesion failure and target lesion revascularisation.

Ethics and dissemination This study has been approved by the Medical Ethics Committee of the region ArnhemNijmegen. The results of this study will be disseminated in a main paper and additional papers with subgroup analyses.

Trial registration number NCT03857971.

\section{Strengths and limitations of this study}

- The PECTUS-obs is the first prospective study to assess the incremental value of optical coherence tomography (OCT) imaging of fractional flow reserve-deferred nonculprit lesions in patients presenting with myocardial infarction (MI).

- OCT is the only imaging modality with a spatial resolution high enough to truly measure fibrous cap thickness, the plaque feature most associated with adverse events.

- In PECTUS-obs, OCT imaging will only be performed at baseline. However, any new MI or revascularisation will be allocated to a specific coronary vessel and lesion by comparison of the baseline and followup angiograms.

- If intracoronary imaging with OCT is able to identify lesions associated with worse outcome, this might warrant studies on focal or pharmacological intervention of OCT-determined vulnerable plaques.

\section{INTRODUCTION}

In patients presenting with myocardial infarction (MI), percutaneous coronary intervention (PCI) of the culprit lesion is the standard method of treatment. ${ }^{1}$ A high percentage of these patients have additional lesions at different sites in the coronary arteries, not responsible for the acute event. The optimal treatment of these nonculprit lesions is subject of extensive research, because their presence confers a greater risk of future major adverse cardiac events (MACE). ${ }^{2}{ }^{3}$ Recent studies showed that complete revascularisation 
results in improved outcomes compared with treatment of the culprit lesion only. ${ }^{4-6}$ However, nonselective revascularisation of all nonculprit lesions may lead to overtreatment.

The selection of nonculprit lesions qualifying for revascularisation is often based on whether a lesion causes ischaemia, as determined by invasive measurements such as the fractional flow reserve (FFR). ${ }^{7}$ In patients with stable coronary artery disease (CAD), FFR-guided complete revascularisation results in better outcomes compared with angiography-guided complete revascularisation. ${ }^{8}$ Nevertheless, the MACE rates at longer term follow-up remain significant in the presence of nonsignificant $\mathrm{CAD} .{ }^{9}$ In patients presenting with $\mathrm{MI}$, this recurrence rate of ischaemic events is even higher. ${ }^{10} \mathrm{~A}$ recent study demonstrated a MACE rate of $23 \%$ in patients with acute coronary syndrome (ACS) versus $11 \%$ in patients with stable CAD at 3.4-year follow-up, after FFR-based deferral of revascularisation. Among these patients with ACS, especially those presenting with non-ST-elevation myocardial infarction (NSTEMI) had a high event rate $(42 \%){ }^{11}$

Apart from coronary physiology, the structural components of nonculprit lesions might provide other markers for future adverse events. Autopsy studies have granted insight into the lesion characteristics that are associated with plaque rupture and subsequent MI or sudden death. These lesions tend to contain a large lipid pool with a thin overlying fibrous cap and display a large degree of outward remodelling. ${ }^{12}{ }^{13}$ These "thin-cap fibroatheromas' (TCFA) are more frequently observed in both culprit and nonculprit lesions of patients presenting with MI than in patients presenting with stable CAD and are a strong predictor of culprit plaque rupture in ACS. ${ }^{14-17}$ Therefore, screening for vulnerable plaques on top of physiological measurements should be evaluated for nonculprit lesions.

Analysis of lesion composition can be performed in vivo with the use of intravascular imaging techniques such as intravascular ultrasound (IVUS), near infrared spectroscopy (NIRS) and optical coherence tomography (OCT). ${ }^{18}$ Prospective studies using IVUS and NIRS showed that identification of lesions at higher risk for future events is feasible. ${ }^{19}{ }^{20}$ However, OCT might prove more suitable for this purpose, due to its specific characteristics. OCT has a 10-20 times higher spatial resolution than IVUS, allowing for better detection of TCFA. Moreover, a complete acquisition of a coronary segment can be provided within a couple of seconds, with a single pullback. Finally, (semi)automated analysis of images is more feasible due to the high resolution of the acquired images. ${ }^{21}$ Nevertheless, OCT has yet to be prospectively validated for its ability to identify lesions at risk for future MACE in patients with MI.

For future studies on potential preventive revascularisation or more aggressive pharmacological therapy in patients with high-risk lesions, prospective studies with clinical outcomes are imperative. In the PECTUS-obs

Table 1 Inclusion and exclusion criteria

\begin{tabular}{ll}
\hline Inclusion criteria & Exclusion criteria \\
\hline Clinical & Clinical \\
Age $\geq 18$ years & Pregnancy. \\
Hospitalisation with a & Haemodynamic instability, \\
STEMI or NSTEMI for & respiratory failure or Killip \\
which patient is subjected & class $\geq 3$ at time of inclusion. \\
to invasive coronary & Previous CABG. \\
angiography (within the & Indication for \\
last 6 weeks). & revascularisation by CABG. \\
& Estimated life expectancy \\
& $<3$ year.
\end{tabular}

Angiographical

- Patient has $\geq 1$ nonculprit, target lesion(s) with following additional characteristics:

- Lesion has visual stenosis of $30 \%-90 \%$.

- Lesion is nonobstructive (FFR $>0.80$ ).

- Lesion is not in-stent restenosis.

CABG, coronary artery bypass grafting; FFR, fractional flow reserve; NSTEMI, non-ST-elevation myocardial infarction; OCT, optical coherence tomography; STEMI, ST-elevation myocardial infarction.

study, we aim to relate OCT-derived plaque characteristics of not significantly flow-limiting, nonculprit lesions to clinical outcome in patients presenting with MI.

\section{METHODS AND ANALYSIS \\ Overview}

The PECTUS-obs study is designed as an international multicentre prospective observational study. Eligible patients have to undergo an index coronary angiography (CAG) during hospitalisation for an acute MI, which reveals one or more nonculprit lesions accessible for imaging with OCT. FFR measurements of these nonculprit lesions are made during the same index procedure or during a staged procedure. Any FFRnonsignificant lesions are subsequently imaged with OCT. Additional criteria are listed in table 1. A total of 438 patients will be included. A flowchart of the study design is depicted in figure 1.

\section{Patients and enrolment}

Patients presenting with MI (ST-elevation and non-STelevation) are screened for potential inclusion in the study. Patients are treated according to the current guidelines for the management of ACS, including referral for CAG and (potential) PCI of the culprit artery. In case of one or more nonculprit lesions of intermediate stenosis (30\%-90\%), clinically indicated FFR measurements are performed in order to determine whether these nonculprit stenoses are haemodynamically significant 


\section{CAG for STEMI / NSTEMI shows a non-culprit lesion}

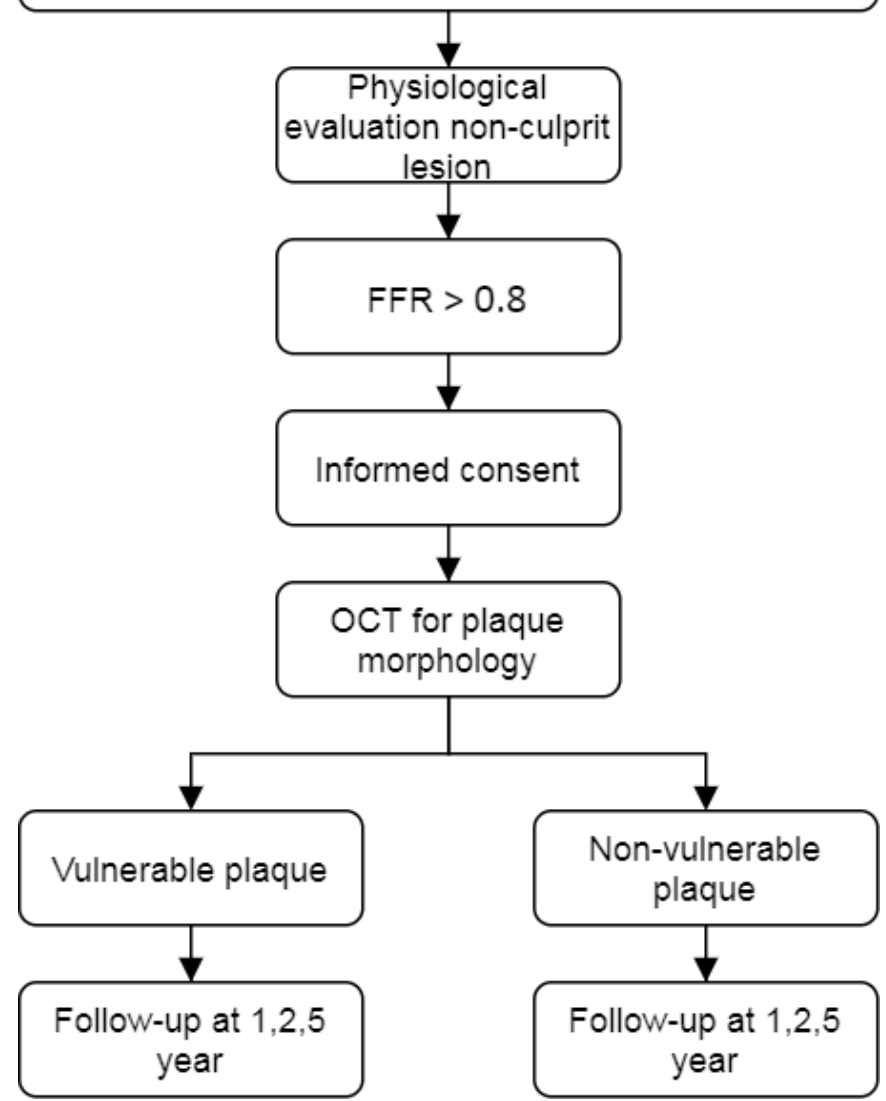

Figure 1 PECTUS-obs flowchart. CAG, coronary angiography, FFR, fractional flow reserve, NSTEMI, nonST-elevation myocardial infarction, OCT, optical coherence tomography, STEMI, ST-elevation myocardial infarction

(figure 2). If a stenosis is nonsignificant $(\mathrm{FFR}>0.8$ ) and the patient is eligible for inclusion based on the criteria listed in table 1, informed consent is obtained for participation in the study. If the FFR is $\leq 0.80$ (haemodynamically significant), the patient is revascularised according to the current therapeutic guidelines.

\section{Timing of FFR measurements and informed consent}

FFR measurements of nonculprit lesions are performed either during the index CAG or during a staged procedure within 6 weeks. If nonculprit lesions are assessed during the index procedure, patients are approached for participation after revascularisation of the culprit artery and any FFR measurements. After oral consent, the OCT pullbacks are performed of all FFR-negative stenoses. Written informed consent is acquired after the procedure. If nonculprit lesion will be evaluated during a staged procedure, written informed consent is acquired prior to the staged procedure.

\section{OCT imaging}

After administration of intracoronary nitrates, an OCT pullback of the target lesion is acquired using the FD-OCT ILUMIEN system (Abbott, USA) over a normal
0.14 " guidewire or pressure wire. The OCT system is CE marked and deployed as intended by the manufacturer. For effective clearing of blood from the imaging field, angiographic contrast media is injected. For the average coronary vessel $14 \mathrm{~mL}$ of contrast media is injected using an automated injector at a rate of $4 \mathrm{~mL} / \mathrm{s}$ at 300 PSI. The contrast amount and/or infusion rate can be adjusted proportionally to coronary artery diameter to ensure good image quality. The segment of interest is scanned with a pull-back speed of $18 \mathrm{~mm} / \mathrm{s}$ ( $54 \mathrm{~mm}$ segment). The entire OCT-pullback is recorded simultaneously with fluoroscopy to ensure that the anatomy of the OCT pullback can be linked to the angiogram. Multiple runs are allowed in case of poor image quality. In case of multiple target lesions eligible for OCT imaging, OCT imaging of each target lesion is performed. OCT images are not used for procedural guidance.

\section{Blood sampling}

During CAG, after OCT imaging is performed, $10 \mathrm{~mL}$ of blood is drawn from the arterial sheath. This blood is used for the determination of biomarkers for plaque or patient vulnerability.

\section{OCT-imaging analysis}

OCT images and corresponding angiograms are analysed offline by trained personnel in an OCT core laboratory. Evaluation of the images is based on tissue characteristics as previously described in OCT expert consensus papers. ${ }^{22}{ }^{23} \mathrm{~A}$ plaque is deemed 'vulnerable' if it contains two of the following characteristics: a lipid arc of $\geq 90^{\circ}$, a cap thickness of $<65 \mu \mathrm{m}$ and either cap rupture or thrombus formation. An example of a vulnerable plaque with a lipid $\operatorname{arc}$ of $>90^{\circ}$ and a cap thickness of $<65 \mu \mathrm{m}$ is shown in figure 2 .

\section{Study endpoints}

The primary study endpoint consists of a composite of major adverse cardiovascular events (all-cause mortality, nonfatal MI (STEMI or NSTEMI) or unplanned revascularisation) at 2-year follow-up in patients with a vulnerable plaque as compared with patients without a vulnerable plaque. Secondary endpoints are: MACE at 1-year and 5-year follow-up, target vessel failure, target vessel revascularisation, target lesion failure and target lesion revascularisation.

\section{Exploratory analyses}

Additional exploratory analyses will be performed by comparing nonculprit plaque characteristics in patients presenting with STEMI versus NSTEMI, in diabetic versus nondiabetic patients, and in male versus female patients. Plaque morphology will also be related to angiographic lesion features. Moreover, in order to accelerate the process of OCT imaging interpretation, automated detection of morphological features associated with MACE will be developed with the use of machine learning. 

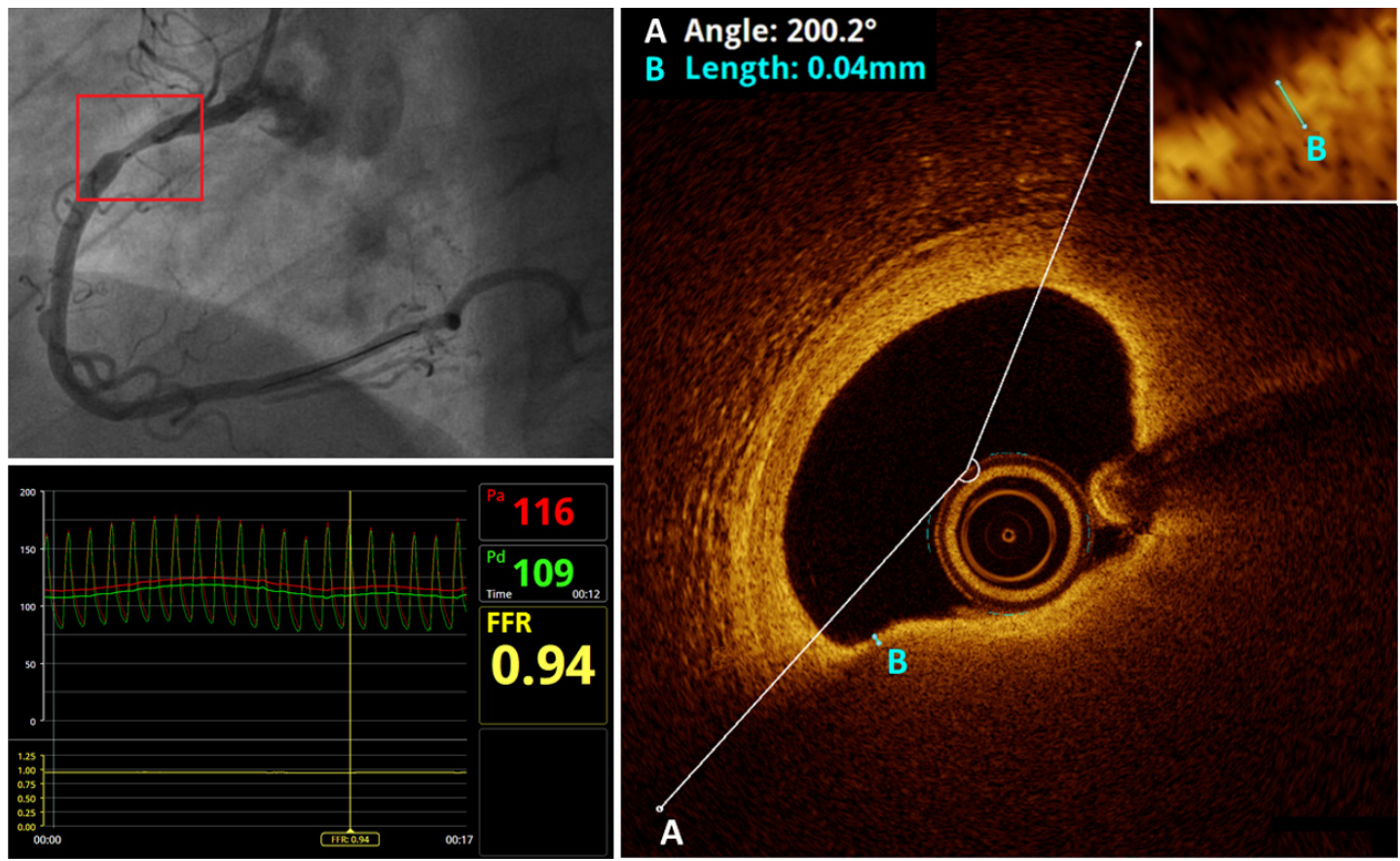

Figure 2 Lesion assessment in the PECTUS-obs study. Upper left: CAG shows a non-culprit lesion (red box) in the proximal RCA. The radiopaque marker inside the vessel at the location of the lesion represents the OCT lens. Lower left: FFR measurement of the lesion reveals that it is nonflow-limiting (FFR=0.94). Right: OCT imaging shows an atherosclerotic plaque with a lipid arc of $200^{\circ}$ and a minimal fibrous cap thickness of $4 \mu \mathrm{m}$. This lesion therefore meets the criteria for a vulnerable plaque. CAG, coronary angiography; FFR, fractional flow reserve; OCT, optical coherence tomography; RCA, right coronary artery.

\section{Follow-up and endpoint adjudication}

At 1-year, 2-year and 5-year patients are followed-up by telephone contact. Medical records (including coronary angiograms) from participating centres, general practitioners and other medical centres are used for the verification of endpoints. Additionally, mortality data are obtained from national registries. A clinical event adjudication committee blinded to OCT data will assess endpoints, separate cardiovascular mortality from noncardiovascular mortality, and allocate any new MI or revascularisation to a specific coronary vessel and lesion by comparison of the baseline and follow-up angiograms.

\section{Sample size calculation and statistical analysis}

The total sample size is calculated at 438 patients. The sample size is calculated to provide $90 \%$ power with a one-sided alpha of 0.025 to identify OCT variables associated with nonculprit lesion-related major adverse cardiovascular events. It is based on the assumption that high-risk OCT-defined vulnerable plaques are identified in $60 \%$ of targeted lesions, on a total event rate of $25 \%$ after 2 years in FFR-deferred lesions in patients with MI, ${ }^{11}{ }^{24}$ and an expected HR of at least 3.5 for OCT defined vulnerable plaques. ${ }^{19}$ A power of $80 \%$ is maintained when the HR is lower than expected, but at least 2.0, or when the event rate is lower than expected but at least $10 \%$. Estimated loss to follow-up is $5 \%$, and inadequate OCT scans prohibiting assessment of vulnerable plaque characteristics are expected in $5 \%$ of cases.
At 2-year follow-up, MACE in patients with vulnerable plaque characteristics will be compared with patients without vulnerable plaque characteristics in terms of HR. Descriptives will be expressed as mean \pm SD (continuous data) or as frequencies and proportions (categorical data). Continuous variables are presented as mean SD if normally distributed or median (IQR) if not normally distributed. Categorical variables are presented as counts and percentages. Continuous variables are compared between groups using the Student t-test or its nonparametric equivalent Mann-Whitney U test. The $\chi^{2}$ test (for comparison of proportions) will be performed where appropriate. Multivariate Cox proportional hazard regression will be used to correct for differences in baseline characteristics like age, sex, diabetes mellitus, hypertension, dyslipidaemia, indication for CAG (STEMI vs NSTEMI), history of MI and history of PCI if necessary. All calculations will be generated by statistical package for social sciences software (SPSS Statistics V.24; IBM, Armonk, New York).

\section{Patient and public involvement}

Patients were not involved in the design of this study.

\section{CURRENT STATUS}

Recruitment commenced in December 2018 and was completed in September 2020. With 2-year follow-up for the primary endpoint, reporting on the study is expected in the beginning of 2023. 


\section{DISCUSSION}

The PECTUS-obs study was designed to investigate the association between OCT-determined characteristics of plaque vulnerability and future MACE in nonflowlimiting, nonculprit lesions of patients presenting with MI.

In current practice, the decision whether or not to preventively treat a nonculprit lesion is primarily based on its physiological significance. Although this strategy is clearly superior in stable $\mathrm{CAD}$, it has yet to be proven in patients presenting with MI. ${ }^{8}$ In STEMI, several large randomised trials have shown that FFR-guided complete revascularisation results in fewer MACE compared with culprit-only revascularisation. ${ }^{25}{ }^{26}$ However, randomised controlled trials directly comparing FFR-guided complete revascularisation with angiography-guided complete revascularisation in STEMI have not yet been conducted, and the only two studies showing a reduction in major clinical endpoints (death and MI) after nonculprit revascularisation were actually guided by angiography rather than physiology. ${ }^{47}$ For patients with NSTEMI, the evidence is even more scarce. In the only available randomised trial, the FAMOUS-NSTEMI trial, MACE rates at 1-year follow-up did not differ between patients with FFR-guided and angiography guided treatment $(8.0 \%$ vs $8.6 \%){ }^{28}$ However, this study was primarily designed to evaluate the effect of FFR measurements on management decisions and was not powered to assess between-group differences in clinical outcomes. The ongoing SLIM trial (NCT03562572) aims to address this gap in knowledge. Nevertheless, even if FFR-guided complete revascularisation proves superior in patients with MI, the long-term MACE rate remains significant. ${ }^{11}$ It, therefore, remains unclear if nonculprit lesion selection based solely on FFR is sufficient, or if other features like plaque morphology need to be taken into account.

In previous prospective intravascular imaging studies, plaque morphology has consistently been analysed using IVUS. In the PROSPECT study, 697 patients with ACS were subjected to three-vessel radiofrequency (RF)IVUS imaging. ${ }^{19}$ All atherosclerotic lesions found in the recordings were subsequently analysed for plaque composition. After a median follow-up of 3.4 years, researchers found that nonculprit lesions with a minimal lumen area (MLA) of $4.0 \mathrm{~mm}^{2}$ or less, a plaque burden of $70 \%$ or greater and those classified as TCFA were associated with a higher rate of MACE. Following PROSPECT, several other studies confirmed the association between RF-IVUS-derived vulnerable plaques and MACE. ${ }^{29} 30$ The main limitation of RF-IVUS when it comes to identifying TCFAs is its poor resolution. In the landmark study by Burke et al, $95 \%$ of ruptured plaques had a fibrous cap thickness of less than $65 \mu \mathrm{m} .{ }^{31}$ More recent reports found that cap thickness of lesions classified as TCFA ranges from $54 \mu \mathrm{m}$ to $84 \mu \mathrm{m} .{ }^{32}$ RF-IVUS has a spatial resolution of approximately $150 \mu \mathrm{m}$, leaving it below the detection range for cap thickness in these lesions. Moreover, of all plaque features that are related with adverse outcomes, cap thickness seems to be the most important. ${ }^{32}$ As mentioned earlier, with a spatial resolution of approximately $10 \mu \mathrm{m}$, we expect that OCT is more suitable for identifying TCFA. However, prospective data on the association between OCT-derived vulnerable plaques and future events are limited. Recently, the arsenal of invasive imaging modalities was broadened by NIRS. The ATHEROREMO-NIRS study proved that NIRS-derived lipid core burden index was associated with MACE at a patient level, whereas the LRP study later expanded on this observation by showing that NIRS can also identify plaques vulnerable to future MACE. ${ }^{20} 33$

The CLIMA study investigated the association between a predefined combination of four high-risk plaque features (MLA $<3.5 \mathrm{~mm}^{2}$, fibrous cap thickness $<75 \mu \mathrm{m}$, a lipid arc $>180^{\circ}$ and the presence of macrophage clusters) and clinical events in patients that underwent OCT imaging of the proximal left anterior descending artery (LAD). ${ }^{34}$ This combination of features proved to be an independent predictor of events with a HR of 7.54. However, this study differed from the current design in several aspects. Even though CLIMA involved prospective follow-up, patients were only included after undergoing OCT imaging for a clinical indication. Moreover, imaging had to be performed on a predefined segment (proximal-mid LAD) that could not include, or be adjacent to, a stenosis of $\geq 50 \%$. Therefore, OCT-imaging, in this study, was used to screen a fixed vessel segment that was relatively free of stenosis, whereas the PECTUS-obs evaluates targeted OCT imaging of angiographically determined stenoses of intermediate severity that are FFR-negative.

The COMBINE study shares more similarities with the current study design. ${ }^{35}$ In this prospective registry of patients with diabetes requiring CAG, OCT imaging of FFR nonflow limiting lesions revealed that patients with TCFAs had increased target lesion-related MACE compared with patients without TCFAs (13.3\% vs $9.7 \%)$ at 18-month follow-up. ${ }^{36}$ In this study, however, only $25 \%$ of included patients had presented with an ACS at baseline.

The current prospective observational study could serve as an important step towards OCT imaging-guided treatment of nonculprit lesions. However, randomised trials need to be conducted in order to evaluate the efficacy of OCT-based interventions. This was attempted in a previous trial in which pre-emptive stenting of FFRnegative OCT-identified vulnerable plaques with ABSORB bioresorbable vascular scaffolds (BVS) was compared with optimal medicinal therapy alone. ${ }^{37}$ Unfortunately this trial was stopped prematurely because the ABSORB BVS was retracted from the market. The currently enrolling PREVENT trial (NCT02316886) also aims to evaluate imaging-guided preemptive stenting, although it uses IVUS and NIRS in addition to OCT. Finally, the recently published PROSPECT ABSORB trial showed good safety outcomes after IVUS/NIRS-guided pre-emptive stenting, while it was not powered for clinical endpoints. ${ }^{38}$ 


\section{CONCLUSION}

The PECTUS-obs is the first prospective study to assess the incremental value of OCT imaging of FFR-deferred nonculprit lesions in patients presenting with MI. If intracoronary imaging with OCT is able to identify lesions associated with worse outcome, this might warrant studies on focal or pharmacological intervention of OCTdetermined vulnerable plaques.

\section{ETHICS AND DISSEMINATION}

This study has been approved by the Medical Ethics Committee of the region Arnhem-Nijmegen (file number 2018-4763). All participants gave informed consent prior to inclusion in the study. The results of this study will be disseminated in a main paper and additional papers with subgroup analyses.

\section{Author affiliations}

${ }^{1}$ Department of Cardiology, Radboudumc, Nijmegen, The Netherlands

${ }^{2}$ Department of Cardiology, Isala Hospitals, Zwolle, The Netherlands

${ }^{3}$ Department of Cardiology, Amphia Hospital, Breda, The Netherlands

${ }^{4}$ Cardiovascular Center, Regional Clinical Hospital, Krasnoyarsk, Russian Federation

${ }^{5}$ Department of Cardiology, North Estonia Medical Centre, Tallinn, Estonia

${ }^{6}$ Department of Cardiology, Meshalkin National Medical Research Center,

Novosibirsk, Russian Federation

${ }^{7}$ Department of Cardiology, Dr Horacio E Oduber Hospital, Oranjestad, Aruba ${ }^{8}$ Department of Cardiology, Albert Schweitzer Hospital, Dordrecht, The Netherlands

${ }^{9}$ Department of Cardiology, Sint Antonius Hospital, Nieuwegein, The Netherlands

${ }^{10}$ Department of Cardiology, Tergooi Hospitals, Blaricum, The Netherlands

${ }^{11}$ Department of Cardiology, Haaglanden Medical Center, Den Haag, The

Netherlands

${ }^{12}$ Department of Cardiology, Zuyderland Medical Center, Heerlen, The Netherlands

${ }^{13}$ Department of Cardiology, Maastricht Universitair Medisch Centrum+, Maastricht, The Netherlands

${ }^{14}$ Department of Cardiology, University Medical Centre Groningen, Groningen, The

Netherlands

${ }^{15}$ Department of Epidemiology, Biostatistics and Health Technology Assessment, Radboudumc, Nijmegen, The Netherlands

Contributors NvR conceived the idea. NvR and JHQM designed the study protocol. ST designed the statistical analyses. JHQM and NvR drafted the manuscript. AB, RHJAV, MM, AVP, PL, OVK, RD, RMO, J-PvK, KA, DJvdH, SR, EL, CC, PD, MAHvL and R-JvG provided critical revisions and substantial intellectual input. All authors agreed with the final version of the manuscript.

Funding This study was financially supported by Abbott Vascular, and Health Holland. Grant numbers are not applicable.

Competing interests None declared.

Patient and public involvement Patients and/or the public were not involved in the design, or conduct, or reporting, or dissemination plans of this research.

Patient consent for publication Not required.

Provenance and peer review Not commissioned; externally peer reviewed.

Open access This is an open access article distributed in accordance with the Creative Commons Attribution Non Commercial (CC BY-NC 4.0) license, which permits others to distribute, remix, adapt, build upon this work noncommercially, and license their derivative works on different terms, provided the original work is properly cited, appropriate credit is given, any changes made indicated, and the use is non-commercial. See: http://creativecommons.org/ licenses/by-nc/4.0/.

\section{ORCID iDs}

Jan-Quinten Mol http://orcid.org/0000-0002-8609-1550

Maarten AH van Leeuwen http://orcid.org/0000-0002-4264-4280
REFERENCES

1 Neumann F-J, Sousa-Uva M, Ahlsson A, et al. 2018 ESC/ EACTS guidelines on myocardial revascularization. Eur Heart $J$ 2019;40:87-165

2 Glaser R, Selzer F, Faxon DP, et al. Clinical progression of incidental, asymptomatic lesions discovered during culprit vessel coronary intervention. Circulation 2005;111:143-9.

3 Goldstein JA, Demetriou D, Grines CL, et al. Multiple complex coronary plaques in patients with acute myocardial infarction. $N$ Engl J Med 2000;343:915-22.

4 Mehta SR, Wood DA, Storey RF, et al. Complete revascularization with multivessel $\mathrm{PCl}$ for myocardial infarction. $\mathrm{N}$ Engl J Med 2019;381:1411-21.

5 Bainey KR, Engstrøm T, Smits PC, et al. Complete vs CulpritLesion-Only revascularization for ST-segment elevation myocardial infarction: a systematic review and meta-analysis. JAMA Cardiol 2020;5:881-888.

6 Rathod KS, Koganti S, Jain AK, et al. Complete versus culprit-only lesion intervention in patients with acute coronary syndromes. J Am Coll Cardiol 2018;72:1989-99.

7 Thim T, van der Hoeven NW, Musto C, et al. Evaluation and management of Nonculprit lesions in STEMI. JACC Cardiovasc Interv 2020;13:1145-54.

8 Tonino PAL, De Bruyne B, Pijls NHJ, et al. Fractional flow reserve versus angiography for guiding percutaneous coronary intervention. N Engl J Med 2009;360:213-24.

9 van Nunen LX, Zimmermann FM, Tonino PAL, et al. Fractional flow reserve versus angiography for guidance of $\mathrm{PCl}$ in patients with multivessel coronary artery disease (FAME): 5-year follow-up of a randomised controlled trial. Lancet 2015;386:1853-60.

10 Masrani Mehta S, Depta JP, Novak E, et al. Association of lower fractional flow reserve values with higher risk of adverse cardiac events for lesions deferred revascularization among patients with acute coronary syndrome. J Am Heart Assoc 2015;4:e002172.

11 Hakeem A, Edupuganti MM, Almomani A, et al. Long-term prognosis of deferred acute coronary syndrome lesions based on nonischemic fractional flow reserve. J Am Coll Cardiol 2016;68:1181-91.

12 Virmani R, Kolodgie FD, Burke AP, et al. Lessons from sudden coronary death: a comprehensive morphological classification scheme for atherosclerotic lesions. Arterioscler Thromb Vasc Biol 2000;20:1262-75.

13 Bentzon JF, Otsuka F, Virmani R, et al. Mechanisms of plaque formation and rupture. Circ Res 2014;114:1852-66.

14 Jang I-K, Tearney GJ, MacNeill B, et al. In vivo characterization of coronary atherosclerotic plaque by use of optical coherence tomography. Circulation 2005;111:1551-5.

15 Kato K, Yonetsu T, Kim S-J, et al. Nonculprit plaques in patients with acute coronary syndromes have more vulnerable features compared with those with non-acute coronary syndromes: a 3-vessel optical coherence tomography study. Circ Cardiovasc Imaging 2012;5:433-40.

16 Maejima N, Hibi K, Saka K, et al. Morphological features of nonculprit plaques on optical coherence tomography and integrated Backscatter intravascular ultrasound in patients with acute coronary syndromes. Eur Heart J Cardiovasc Imaging 2015;16:190-7.

17 lannaccone M, Quadri G, Taha S, et al. Prevalence and predictors of culprit plaque rupture at OCT in patients with coronary artery disease: a meta-analysis. Eur Heart $J$ Cardiovasc Imaging 2016;17:1128-37.

18 Bom MJ, van der Heijden DJ, Kedhi E, et al. Early detection and treatment of the vulnerable coronary plaque: can we prevent acute coronary syndromes? Circ Cardiovasc Imaging 2017;10.

19 Stone GW, Maehara A, Lansky AJ, et al. A prospective natural-history study of coronary atherosclerosis. N Engl J Med 2011;364:226-35.

20 Waksman R, Di Mario C, Torguson R, et al. Identification of patients and plaques vulnerable to future coronary events with near-infrared spectroscopy intravascular ultrasound imaging: a prospective, cohort study. Lancet 2019;394:1629-37.

21 Kini AS, Vengrenyuk Y, Yoshimura T, et al. Fibrous Cap Thickness by Optical Coherence Tomography In Vivo. J Am Coll Cardiol 2017;69:644-57.

22 Prati F, Regar E, Mintz GS, et al. Expert review document on methodology, terminology, and clinical applications of optical coherence tomography: physical principles, methodology of image acquisition, and clinical application for assessment of coronary arteries and atherosclerosis. Eur Heart J 2010;31:401-15.

23 Tearney GJ, Regar E, Akasaka T. Consensus standards for acquisition, measurement, and reporting of intravascular optical coherence tomography studies: a report from the International Working group for intravascular optical coherence tomography standardization and validation. J Am Coll Cardiol 2012;59:1058-72. 
24 Sels J-WEM, Tonino PAL, Siebert U, et al. Fractional flow reserve in unstable angina and non-ST-segment elevation myocardial infarction experience from the fame (fractional flow reserve versus angiography for multivessel evaluation) study. JACC CardiovasC Interv 2011;4:1183-9.

25 Engstrøm T, Kelbæk H, Helqvist S, et al. Complete revascularisation versus treatment of the culprit lesion only in patients with STsegment elevation myocardial infarction and multivessel disease (DANAMI-3-PRIMULTI): an open-label, randomised controlled trial. Lancet 2015;386:665-71.

26 Smits PC, Abdel-Wahab M, Neumann F-J, et al. Fractional flow reserve-guided multivessel angioplasty in myocardial infarction. $N$ Engl J Med 2017;376:1234-44.

27 Wald DS, Morris JK, Wald NJ, et al. Randomized trial of preventive angioplasty in myocardial infarction. $N$ Engl $J$ Med 2013;369:1115-23.

28 Layland J, Oldroyd KG, Curzen N, et al. Fractional flow reserve vs. angiography in guiding management to optimize outcomes in non-ST-segment elevation myocardial infarction: the British heart Foundation FAMOUS-NSTEMI randomized trial. Eur Heart $J$ 2015;36:100-11.

29 Calvert PA, Obaid DR, O'Sullivan M, et al. Association between IVUS findings and adverse outcomes in patients with coronary artery disease: the VIVA (VH-IVUS in vulnerable atherosclerosis) study. JACC Cardiovasc Imaging 2011;4:894-901.

30 Cheng JM, Garcia-Garcia HM, de Boer SPM, et al. In vivo detection of high-risk coronary plaques by radiofrequency intravascular ultrasound and cardiovascular outcome: results of the ATHEROREMO-IVUS study. Eur Heart J 2014;35:639-47.
31 Burke AP, Farb A, Malcom GT, et al. Coronary risk factors and plaque morphology in men with coronary disease who died suddenly. N Engl $J$ Med 1997;336:1276-82.

32 Narula J, Nakano M, Virmani R, et al. Histopathologic characteristics of atherosclerotic coronary disease and implications of the findings for the invasive and noninvasive detection of vulnerable plaques. $J$ Am Coll Cardiol 2013;61:1041-51.

33 Schuurman A-S, Vroegindewey M, Kardys I, et al. Near-Infrared spectroscopy-derived lipid core burden index predicts adverse cardiovascular outcome in patients with coronary artery disease during long-term follow-up. Eur Heart J 2018;39:295-302.

34 Prati F, Romagnoli E, Gatto L, et al. Relationship between coronary plaque morphology of the left anterior descending artery and 12 months clinical outcome: the CLIMA study. Eur Heart $J$ 2020;41:383-91.

35 Kennedy MW, Fabris E, ljsselmuiden AJ, et al. Combined optical coherence tomography morphologic and fractional flow reserve hemodynamic assessment of non- culprit lesions to better predict adverse event outcomes in diabetes mellitus patients: combine (OCT-FFR) prospective study. rationale and design. Cardiovasc Diabetol 2016;15:144.

36 Kedhi E. Combined optical coherence tomography and fractional flow reserve assessment to better predict adverse event outcomes in DM patients: combine (OCT-FFR) trial. Transcatheter Cardiovascular Therapeutics Annual Conference; 14 Oct, 2020.

37 Mol J-Q, Bom MJ, Damman P, et al. Pre-emptive OCT-Guided angioplasty of vulnerable intermediate coronary lesions: results from the prematurely halted PECTUS-Trial. J Interv Cardiol 2020;2020:1-8.

38 Stone GW, Maehara A, Ali ZA, et al. Percutaneous coronary intervention for vulnerable coronary atherosclerotic plaque. J Am Coll Cardiol 2020;76:2289-301. 